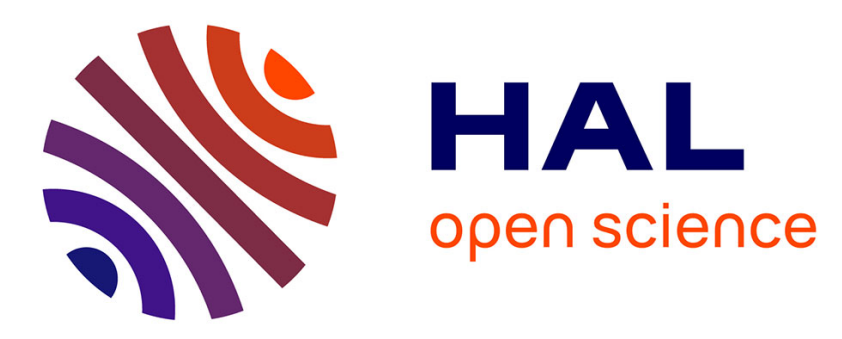

\title{
Statistical analysis of cloud light scattering and microphysical properties obtained from airborne measurements
}

Olivier Jourdan, Sergey Oshchepkov, Jean-François Gayet, Valery

Shcherbakov, Harumi Isaka

\section{To cite this version:}

Olivier Jourdan, Sergey Oshchepkov, Jean-François Gayet, Valery Shcherbakov, Harumi Isaka. Statistical analysis of cloud light scattering and microphysical properties obtained from airborne measurements. Journal of Geophysical Research, 2003, 108 (D5), 10.1029/2002jd002723 . hal-01982541

\section{HAL Id: hal-01982541 https://hal.science/hal-01982541}

Submitted on 15 Jan 2019

HAL is a multi-disciplinary open access archive for the deposit and dissemination of scientific research documents, whether they are published or not. The documents may come from teaching and research institutions in France or abroad, or from public or private research centers.
L'archive ouverte pluridisciplinaire HAL, est destinée au dépôt et à la diffusion de documents scientifiques de niveau recherche, publiés ou non, émanant des établissements d'enseignement et de recherche français ou étrangers, des laboratoires publics ou privés. 


\title{
Statistical analysis of cloud light scattering and microphysical properties obtained from airborne measurements
}

\author{
Olivier Jourdan, Sergey Oshchepkov, and Jean-Francois Gayet \\ Laboratoire de Météorologie Physique, CNRS, Université Blaise Pascal, Clermont-Ferrand, France
}

Valery Shcherbakov

Stepanov Institute of Physics, National Academy of Sciences, Minsk, Belarus

Harumi Isaka

Laboratoire de Météorologie Physique, CNRS, Université Blaise Pascal, Clermont-Ferrand, France

Received 4 July 2002; revised 7 November 2002; accepted 18 December 2002; published 8 March 2003.

[1] A new statistical analysis of the in situ scattering phase function measurements performed by the Laboratoire de Météorologie Physique's airborne polar nephelometer is implemented. A principal component analysis along with neural networks leads to the classification of a large data set into three typical averaged scattering phase functions. The cloud classification in terms of particle phase composition (water droplets, mixed-phase, and ice crystals) is done by a neural network and is validated by direct Particle Measuring Systems, Inc., probe measurements. The results show that the measured scattering phase functions carry enough information to accurately retrieve component composition and particle size distributions. For each classified cloud, we support the statement by application of an inversion method using a physical model of light scattering to the average scattering phase function. Furthermore, the retrievals are compared with size composition obtained by independent direct measurements. INDEX TERMS: 0320

Atmospheric Composition and Structure: Cloud physics and chemistry; 0649 Electromagnetics: Optics; 3260 Mathematical Geophysics: Inverse theory; 3359 Meteorology and Atmospheric Dynamics: Radiative processes; 3394 Meteorology and Atmospheric Dynamics: Instruments and techniques; KEYWORDS: statistical analysis of geophysical data, inverse problem, characterization of cloud optical and microphysical properties, in situ cloud measurements, neural network classification

Citation: Jourdan, O., S. Oshchepkov, J.-F. Gayet, V. Shcherbakov, and H. Isaka, Statistical analysis of cloud light scattering and microphysical properties obtained from airborne measurements, J. Geophys. Res., 108(D5), 4155, doi:10.1029/2002JD002723, 2003.

\section{Introduction}

[2] Microphysical and optical properties of cloud particles are known to be a primordial source of information for the understanding of light interactions in the atmosphere system through radiative and chemical processes, hence of the cloud feedback of earth climate. Well-documented observations of the scattering properties for different types of clouds are required for reliable modeling of radiative transfer characteristics in a cloudy atmosphere (see, among others Kinne et al. [1992]) and to improve satellite and ground-based cloud product retrievals (see, among others, Rossow and Schiffer [1991]). The main goal of this study is to draw up a set of representative scattering phase functions and particle size distributions for different types of clouds relative to their particle-phase composition (liquid-water phase, solid-water phase: i.e. ice crystals, and mixed phase: i.e. water droplets and ice crystals) using a statistical analysis of around 60,000 measurements. A physical modeling of particle scattering properties supports the interpretation of the statistical results.

Copyright 2003 by the American Geophysical Union. 0148-0227/03/2002JD002723\$09.00
Various intensive aircraft field measurements using an airborne polar nephelometer and PMS probes allowed us to study simultaneous observations of cloud optical and microphysical properties. The data have been obtained during three campaigns, namely: ARAT'97 [Duroure et al., 1998], CIRRUS'98 [Durand et al., 1998], JACCS'99 [Asano et al., 2002; Gayet et al., 2002], which were carried out from Clermont-Ferrand (central part of France), Tarbes (SouthWest of France), and over the Sea of Japan, respectively. Collectively, these campaigns present the advantage of including a large set of data obtained in a wide variety of meteorological conditions.

[3] This paper investigates the potential of statistical analysis of the in situ scattering phase functions performed by the polar nephelometer [Gayet et al., 1998] for the description of clouds' optical and microphysical properties. First, we describe the implementation of a principal component analysis (PCA) on the data to find pertinent relationships amongst scattering phase functions of different cloud compositions. Then, we present the classification of the patterns we evidenced, by using neural networks leading to the determination of typical optical characteristics. Finally, from the established typical scattering phase functions, the 
inverse method of Oshchepkov et al. [2000] for the retrieval of particle size distribution and composition is applied for the validation of the results.

\section{Statistical Data Analysis}

\subsection{Description of the Probes and Data Processing}

[4] The ARAT'97 and JACCS'99 experiments involve observations in stratiform water and mixed-phase clouds whereas cirrus clouds were sampled during the CIRRUS'98 campaign. These data represent more than 60,000 optical and microphysical measurements with a sampling frequency of $1 \mathrm{~Hz}$. This corresponds to measurements sampled with a spatial resolution of about $100 \mathrm{~m}$ according to the mean cruising speed of the aircraft. The aircraft were equipped with the polar nephelometer for the scattering phase function measurements, a Particle Measuring Systems, Inc., (PMS) 2D-C probe for measurements of particle images and, except for CIRRUS' 98 , a PMS FSSP100 probe for droplet size distribution measurements.

[5] The polar nephelometer [Gayet et al., 1998] measures the scattering phase function of an ensemble of cloud particles (from a few micrometers to about $800 \mu \mathrm{m}$ diameter), which intersect a collimated laser beam near the focal point of a parabolic mirror. The laser beam is provided by a high-power $(0.8 \mathrm{~W})$ multimode laser diode operating at a wavelength of $804 \mathrm{~nm}$. The light scattered at polar angles from $\pm 3.49^{\circ}$ to $\pm 169^{\circ}$ is reflected onto a circular array of 56 photodiodes. Nevertheless, the measurements at small forward scattering angles are not available due to contamination by the diffracted light from the edges of holes drilled on the polar nephelometer paraboloidal mirror. Consequently, only 34 channels corresponding to scattering angles ranging from $\pm 15^{\circ}$ to $\pm 169^{\circ}$ are exploitable.

[6] The bi-dimensional optical array spectrometer (PMS 2D-C) provides information on the crystal size and shape with a size range from $25 \mu \mathrm{m}$ to $800 \mu \mathrm{m}$ by recording cloud particles diffraction images. The PMS Forward Scattering Spectrometer Probe (FSSP-100) is an optical particle counter for the sampling of droplet size-distribution from $3 \mu \mathrm{m}$ to $45 \mu \mathrm{m}$ in diameter. The data processing method of the above instruments has already been described in detail by Auriol et al. [2001] and Gayet et al. [2002].

[7] In order to discriminate the cloud phase composition we define a criterion $\left(\mathrm{R}_{\text {mic }}\right)$, which is determined by the bulk quantities derived from the particle size distributions measured by both the 2D-C and FSSP-100 probes:

$$
R_{\text {mic }}=L W C / I W C
$$

where LWC is the liquid water content $\left(\mathrm{g} \mathrm{m}^{-3}\right)$ derived from the FSSP-100 and IWC refers to the ice water content $\left(\mathrm{g} \mathrm{m}^{-3}\right)$ derived from 2D-C measurements. A comparison between side scattering angles behavior of a large set of experimental phase functions in various cloud situations and the ratio $R_{\text {mic }}$ led us to define empirical thresholds to distinguish the cloud phase. As a matter of fact, the side scattering angles are very sensitive to the presence (or not) of ice crystal particles [Gayet et al., 2002]. In order to remove from the data set the cloud portions with very low particle concentration, a particle density threshold has been added. The FSSP-100 and 2D-C threshold concentrations of $1 \mathrm{~cm}^{-3}$ and $11^{-1}$ (i.e. $0.001 \mathrm{~cm}^{-3}$ ), respectively, roughly correspond to the lower representative values which can be measured by these probes. Table 1 summarizes these criteria for cloud phase classification. This method could be connected to the methodology proposed by Cober et al. [2001] which assesses the relative ice and liquid quantities using the responses from six available airborne microphysical instruments. Although our method is less sophisticated than the one developed by Cober et al. [2001], it proves to be reliable enough to identify liquid, mixed and glaciated cloud conditions. It should be noticed that the previously established microphysical criteria $\left(\mathrm{R}_{\text {mic }}\right.$ and concentration thresholds) will also be used to validate the neural network classification of the scattering phase functions, as discussed in section 3 .

\subsection{Application of the Principal Component Analysis}

[8] In this section, a Principal Component Analysis (PCA) is applied to a large set of optical measurements (scattering phase functions) obtained during the campaigns listed in section 2.1.

[9] A PCA is designed to generate a new set of uncorrelated parameters, called principal components, representative of the original data set. Algebraically, principal components could be defined as particular linear combinations of a set of variables. These linear combinations represent the selection of a new coordinate system obtained by rotating the original system of coordinates. The new axes correspond to the directions with maximum variability and provide a simpler description of the covariance structure of the original set of variables (see, among others, Johnson and Wichern [1998]). In many practical implementations, only a few principal components are required to reproduce almost all the system's variability with reasonably good accuracy. This is especially true when a limited number of primary physical parameters have a major impact on the measured functions (in this case, scattering phase functions).

[10] The main objectives of this section are data reduction, denoising and possible physical interpretation of the revealed patterns. Besides, PCA serves as an intermediate step to rearrange the data set before the cloud classification performed in section 2.3. Indeed, most of the classification approaches like cluster analysis encounter great difficulties when the number of variables is large and when there is high correlation among them.

[11] In order to apply PCA to the optical data set, we follow the well-established methodology (see, among others, Legendre and Legendre [1998]) expanding the optical measurements in terms of eigenvectors of the data set correlation matrix. A particular feature of this analysis consists in making the calculations in the logarithmic space. The logarithm of nonnegative values is used in our application of the PCA in order to make comparable the magnitudes of all variables. Otherwise, the first principal components would stand only for the characteristics having highest variance and would not reproduce all the system variability. At the same time, the logarithm is also a monotonous function, so the features of the scattering phase functions (optical measurements) can be estimated from the properties of their logarithm.

[12] Accordingly, to parameterize the measured functions, we expand the vectors $\mathbf{f}_{\mathbf{j}}=\log \left[\boldsymbol{\sigma}_{\mathbf{j}}(\theta)\right]$ in terms of eigenvectors of the correlation matrix of the logarithm of scattering 
Table 1. Cloud Classification in Terms of Particle Composition According to Bulk Quantities $\left(\mathrm{R}_{\text {mic }}\right)$ and Particle Density Criteria ${ }^{\mathrm{a}}$

\begin{tabular}{lccc}
\hline & $\mathrm{R}_{\text {mic }}>0.5$ ConcFssp $>1 \mathrm{~cm}^{-3}$ & $0.5 \geq \mathrm{R}_{\mathrm{mic}} \geq 0.1$ ConcFssp $>1 \mathrm{~cm}^{-3}$ and Conc2dc $>11^{-1}$ & $\mathrm{R}_{\mathrm{mic}}<0.1 \mathrm{Conc} 2 \mathrm{dc}>101^{-1}$ \\
\hline $\begin{array}{l}\text { Classification } \\
\text { Description }\end{array}$ & $\begin{array}{c}\text { Liquid Phase Clouds } \\
\text { water dominating }\end{array}$ & $\begin{array}{c}\text { Mixed Phase Clouds } \\
\text { water + ice }\end{array}$ & $\begin{array}{c}\text { Solid Phase Clouds } \\
\text { ice dominating }\end{array}$ \\
\hline
\end{tabular}

${ }^{\mathrm{a}}$ ConcFssp represents the number concentration derived from the FSSP-100 probe and Conc2dC is the number concentration measured by the 2D-C probe.

phase functions $\sigma_{\mathbf{j}}(\theta)\left(\sigma_{\mathbf{j}}(\theta)\right.$ is the non-normalized angular scattering phase function of the jth measurement and $\theta$ is the scattering angle). It should be noted that PCA is particularly adequate when there is a fairly high linear correlation among measured parameters of a data set.

[13] Figure 1a represents the first four eigenvectors, $\xi_{\mathrm{I}}$ $(1=1,2,3,4)$, of the correlation matrix of $\mathbf{f}_{\mathbf{j}}$ calculated for our data set (i.e. about 60,000 measurement points), as a function of scattering angle $\theta$. The results show the presence of peaks at some particular scattering angles pointed out by the black arrows $\left(\theta=32.5^{\circ}, 53.5^{\circ}, 64^{\circ}, 134^{\circ}, 162^{\circ}\right.$ and $165^{\circ}$ ). The high discontinuities of the eigenvectors reveal the low correlation of the corresponding scattering angles with the other ones. Such result leads to the conclusion that the data at those angles are not reliable. Consequently, this type of analysis enables us to detect the polar nephelometer channels that are not working accurately and which will not be used in the following.

[14] After excluding the unreliable data, the correlation matrix and its eigenvectors were computed another time. The values of the first four normalized eigenvalues $\lambda_{1}$ corresponding to the first four eigenvectors, $\xi_{1}(1=1,2,3,4)$ are reported on Figure 1b. (with the same presentation as on Figure 1a). The first eigenvector represents $97 \%$ of the total population variance, the three next ones $1.1 \%, 0.6 \%$ and $0.3 \%$, respectively. These four values add up to $99 \%$ of the total sum of the eigenvalues, this means that $99 \%$ of the variations of vectors $\sigma_{\mathrm{j}}$ can be described by the following expression:

$$
\boldsymbol{f}_{j}(\boldsymbol{\theta})=\langle\boldsymbol{f}(\boldsymbol{\theta})\rangle+\sum_{l=1}^{4} C_{j, l} \xi_{l}(\theta)
$$

where $\langle\mathbf{f}(\boldsymbol{\theta})\rangle$ represents the average phase function of the total data set and $C_{j, 1}=\left(\mathbf{f}_{\mathbf{j}}-\langle\mathbf{f}(\boldsymbol{\theta})\rangle\right)^{\mathrm{T}}$. $\boldsymbol{\xi}_{\mathbf{l}}(\boldsymbol{\theta})$ are the expansion coefficients of $\mathbf{f}_{\mathbf{j}}\left({ }^{\mathrm{T}}\right.$ designates a transposed matrix). The first four coefficients $\mathrm{C}_{\mathrm{j}, 1}$ contain the major part of the information about a particular scattering phase function. Consequently, in our case, the PCA leads to a significant reduction of the dimensionality of the data set. Instead of the 28-dimensional space of scattering phase functions (after removing the unreliable channels), we have the 4dimensional space of the expansion coefficients $\mathrm{C}_{\mathrm{j}, 1}$. In principle, each observation can be expressed with good accuracy as a linear combination of the selected set of eigenvectors $\xi_{1}, \xi_{2}, \xi_{3}, \xi_{4}$ by equation (2).

[15] Some important information may be obtained from an analysis of the first four eigenvectors behaving. The first eigenvector $\xi_{1}$ is almost constant, meaning that $97 \%$ of the phase function variations are caused by changing the particle concentration. The second eigenvector $\xi_{2}$ reverses sign twice, at the angles of $34^{\circ}$ and $125^{\circ}$. Accordingly, it means that increasing the sideward scattering is followed by decreasing energy scattered into smaller $\left(15^{\circ}<\theta<34^{\circ}\right)$ and higher angles $\left(125^{\circ}<\theta<155^{\circ}\right)$, and vice versa. At this point one can see that the PCA results are in total agreement with the general light scattering theory. Here, the most important information consists in the percentage of the phase function variations (the values of $\lambda_{1}$ and $\lambda_{2}$ ).

[16] The third eigenvector, $\xi_{3}$, reverses sign at $21^{\circ}, 75^{\circ}$, and $137^{\circ}$ and should carry information about the particle phase composition because the angle interval $\left[75^{\circ}-137^{\circ}\right]$ is quite close to that one of $\left[60^{\circ}-140^{\circ}\right]$. It is well known that the scattering properties in sideward angles, approximately between $60^{\circ}$ and $140^{\circ}$, are sensitive to the particle shape and structure [Yang and Liou, 1996; Doutriaux-Boucher et al., 2000]. Comparatively to the particles in a liquid-water phase, the mixed phase and the ice phase particles scatter more energy in the angle range from $75^{\circ}$ to $137^{\circ}$ and from $15^{\circ}$ to $21^{\circ}$. On the other hand, they scatter less energy for $21^{\circ}<\theta<75^{\circ}$.

[17] Finally, the eigenvector $\xi_{4}$ is close to zero, except for $\theta<44^{\circ}$ and $\theta>110^{\circ}$. Its sign is opposite for these two intervals. In principle, such features could be connected with some scattering properties that affect the asymmetry parameter. But, these explanations should be considered as an zero-order interpretation because there is no one-to-one correspondence between the principal components and some physical features.

[18] As an example, Figure 1c illustrates the different steps to restore a particular scattering phase function observation using equation (2). The corresponding logarithm of measured phase function is represented by open circle symbols and the average logarithm of scattering phase function of the total data set is plotted with full circle symbols. By adding the contributions of the first four principal components, the restored logarithm of scattering phase function (thick grey curve) is found to be in very good agreement with the measured one (with a root mean square error of $6 \%$ ). The vectors $\mathbf{P C}_{\mathbf{i}}$ presented on the Figure 1c are defined by:

$$
\mathbf{P C}_{\mathbf{i}}=\langle f(\theta)\rangle+\sum_{l=1}^{i} C_{j, l} \xi_{l}(\theta)
$$

[19] The first principal component obviously contributes to most of variability (thin black curve) and its magnitude depends on the particle number density. In this particular case, the contribution of the second principal component is relatively small (see differences between dotted black and thin black curves). At the same time, the contribution of the third component is noticeable. The thick black line almost fits to the measured data. As it was expected, the contribution of the fourth component shows up a better fitting at the left and right ends of the phase function.

\subsection{Cloud Phase Classification Using Neural Networks}

[20] The next step is to perform a cloud classification of the measured cloud phase function in terms of particle phase composition (water-droplets, mixed-phase and ice-crystals). 


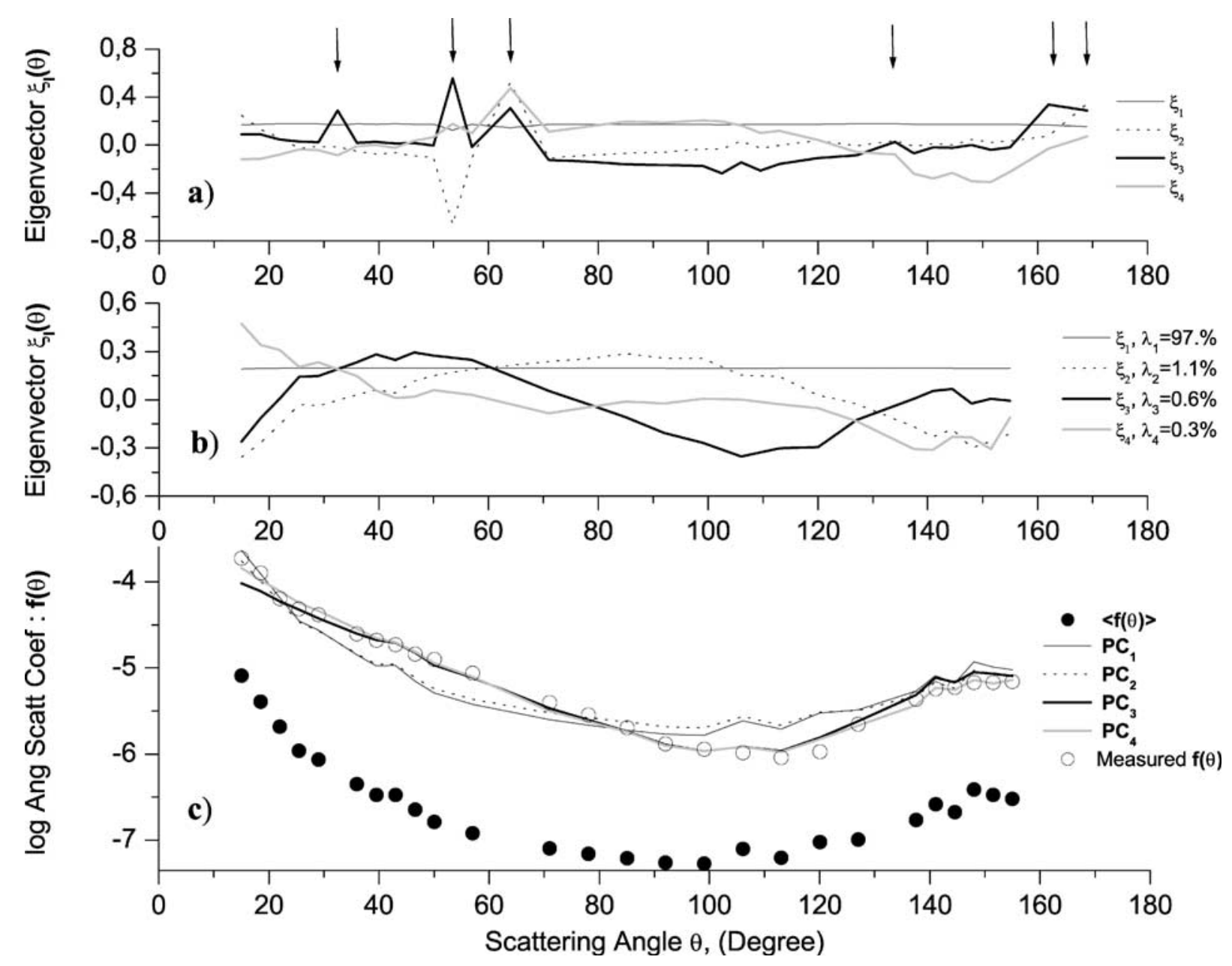

Figure 1. Results of the Principal Component Analysis applied on a data set of 60,000 measurements obtained with the polar nephelometer in various cloud situations. (a) First four eigenvectors of the scattering phase function correlation matrix versus the 34 measured scattering angles). The black arrows indicate channels that are not working correctly. (b) Same as (a). Here, only the 28 reliable channels are reported. The values of the first four normalized eigenvalues $\lambda_{1}$ of $\xi_{1}$ are displayed. (c) Summed contributions, up to a given principal component $\left(\mathbf{P C}_{\mathbf{i}}\right)$ for one example of restored scattering phase function (see text).

This classification stems from the interpretation of the expansion coefficients $\mathrm{C}_{\mathrm{j}, 1}(1=1,2,3,4)$. The typical clusters are shown on the diagram $\left(\mathrm{C}_{\mathrm{j}, 1}\right.$ versus $\mathrm{C}_{\mathrm{j}, 3}$, see Figure 2$)$ where the scattering phase functions are linked to the cloud's composition through their coefficients $C_{j, 1}$ and $C_{j, 3}$.

[21] Different classification techniques (clustering analysis and competitive neural networks) were tested but the results were not satisfactory. Finally, a feed-forward perceptron with two hidden layers using supervised training was chosen to analyze relationships among the first four principal components. Each layer is characterized by its weight matrix $\mathbf{W}$, bias vector $\mathbf{b}$ and output vector $\mathbf{a}$. The input vector $\left(\mathbf{a}_{\mathrm{k}-1}\right)$ and output vector $\left(\mathbf{a}_{\mathrm{k}}\right)$ of the kth layer are interconnected as:

$$
\mathbf{a}_{\mathrm{k}}=\mathrm{T}^{\mathrm{k}}\left(\mathbf{x}_{\mathrm{k}}\right) \text { with } \mathbf{x}_{\mathrm{k}}=\mathbf{W}_{\mathrm{k}} \mathbf{a}_{\mathrm{k}-1}+\mathbf{b}_{\mathrm{k}}
$$

where $\mathrm{T}^{\mathrm{k}}$ is the transfer function (the hyperbolic tangent in our case) of the kth layer. The training set is composed of M pairs: $\left(\mathbf{a}_{0 \mathrm{~m}}, \mathbf{s}_{\mathrm{m}}\right) \mathrm{m}=1, \ldots, \mathrm{M}$, where $\mathbf{a}_{0 \mathrm{~m}}$ and $\mathbf{s}_{\mathrm{m}}$ designate the $\mathrm{mth}$ input and target vectors. All the weight matrixes and biases are determined by minimizing a performance function $\mathrm{E}$ as:

$$
E=\sum_{m=1}^{M}\left(\mathbf{a}_{2 \mathbf{m}}-\mathbf{s}_{\mathbf{m}}\right)^{T} \cdot\left(\mathbf{a}_{2 \mathbf{m}}-\mathbf{s}_{\mathbf{m}}\right)
$$

[22] In our case, the input vectors consist of the first four expansion coefficients $\mathrm{C}_{\mathrm{j}, 1}(1=1, \ldots, 4)$ and the output vectors define the class (water, mixed phase, ice) of which an input vector belongs to. The training set is composed of patterns representing $5 \%$ of the total data set. Pairs are randomly selected from the data, which we are sure to belong to a particular class. The neural network is trained using the Levenberg-Marquardt algorithm for the back-propagation with MacKay's Bayesian regularization [MacKay, 1992]. The MATLAB neural network toolbox was used. Finally, the generalization phase is performed with the entire data set.

\section{Results of the Statistical Analysis}

[23] The principal component analysis has been applied to our data set, which includes both cloud events and 'clear sky' segments. These 'clear sky' events are mostly charac- 


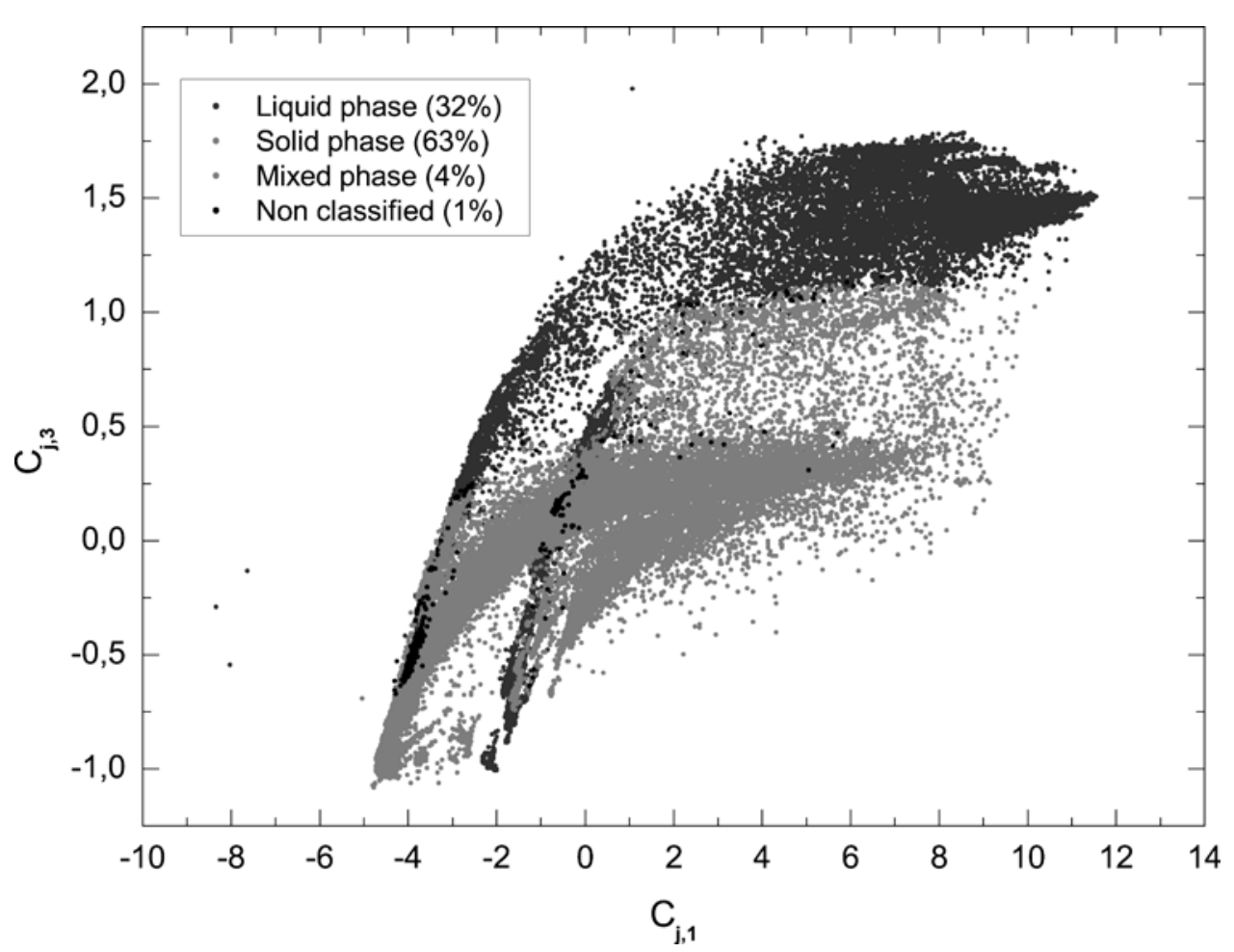

Figure 2. Expansion coefficient diagram: First coefficient $\left(C_{j, 1}\right)$ versus the third coefficient $\left(C_{j, 3}\right)$. The clusters are colored (blue, red and olive green) according to the cloud composition (water droplet clouds, mixed-phase clouds and ice particles respectively). See color version of this figure at back of this issue.

terized by a noise signal on the polar nephelometer measurements (offset), which can be different according to the experiment under consideration because it strongly depends on the instrument's optical settings. High concentrations of aerosols could also contribute to the variation of the signal measured by the polar nephelometer.

[24] The scatter plot of the $C_{j, 1}$ expansion coefficient (corresponding to the first eigenvector $\xi_{1}$ ) versus the $C_{j, 3}$ coefficient, on Figure 2, describes the optical features of the clouds in the clearest representation. Actually, the highest values of $\mathrm{C}_{\mathrm{j}, 3}$ coefficient correspond to the lowest side scattering energies and to the highest scattering in the angular range of $35^{\circ}$ to $60^{\circ}$. This means that high values of $\mathrm{C}_{\mathrm{j}, 1}$ and $\mathrm{C}_{\mathrm{j}, 3}\left(\mathrm{C}_{\mathrm{j}, 1}>5\right.$ and $\left.\mathrm{C}_{\mathrm{j}, 3}>1\right)$ correspond to liquid phase clouds with high concentration of water droplets whereas low values of $C_{j, 3}\left(C_{j, 3}<0.45\right)$ indicate the presence of ice crystals with a large side scattering behavior. The results of neural network classification, determined from the scattering behavior of the cloud particles, clearly show three clusters specific of the particle phase. On Figure 2 , the blue cluster represents water droplet clouds $(32 \%$ of the measurements according to our cloud phase classification), the olive green cluster $(63 \%)$ indicates ice particle clouds and mixed-phase clouds $(4 \%)$ correspond to the red cluster. The data points that have a $\mathrm{C}_{\mathrm{j}, 1}$ value lower than zero will not be considered in the following. As a matter of fact, these points correspond to clouds with a small concentration of particles (low value of signal to noise ratio) or to clear sky conditions. The variability of the $\mathrm{C}_{\mathrm{j}, 3}$ component could be due to aerosol contribution and/or to the variations of the clear sky noise signal.
[25] In order to compare and validate the cluster classification considered above, an alternative classification is presented according to our cloud phase criteria in Table 1, based on direct microphysical measurements from the PMS instruments. The different cloud compositions are displayed in Figure 3 with same color scheme as Figure 2. The results in Figure 2 and the direct classification displayed in Figure 3 are in good agreement, as expected. The black dots $(50 \%$ of the measurements) represent the clear sky parts and are mainly observed for values of $\mathrm{C}_{\mathrm{j}, 1}$ and $\mathrm{C}_{\mathrm{j}, 3}$ lower than zero. This analysis based on microphysical measurements is, however, still limited, mainly because of the high uncertainties on microphysical parameters derived from the PMS probe [Gayet et al., 2002] but also because the criteria given by equation (1) does not involve enough information about cloud composition. This classification technique involves a limited amount of parameters to characterize the cloud phase (ConcFssp, Conc2dC, IWC, LWC) thus presenting a serious disadvantage in comparison to the classification based on a PCA along with neural networks. Indeed, the latter offers the possibility to take into account a scattering phase function on 28 angles to discriminate cloud phase.

\section{Interpretation of Representative Scattering Phase Functions Using Physical Modeling}

[26] In this section, we compare particle size distributions retrieved from average scattering phase functions with those averaged from direct PMS microphysical measurements for each cloud type. This is an important issue for further 


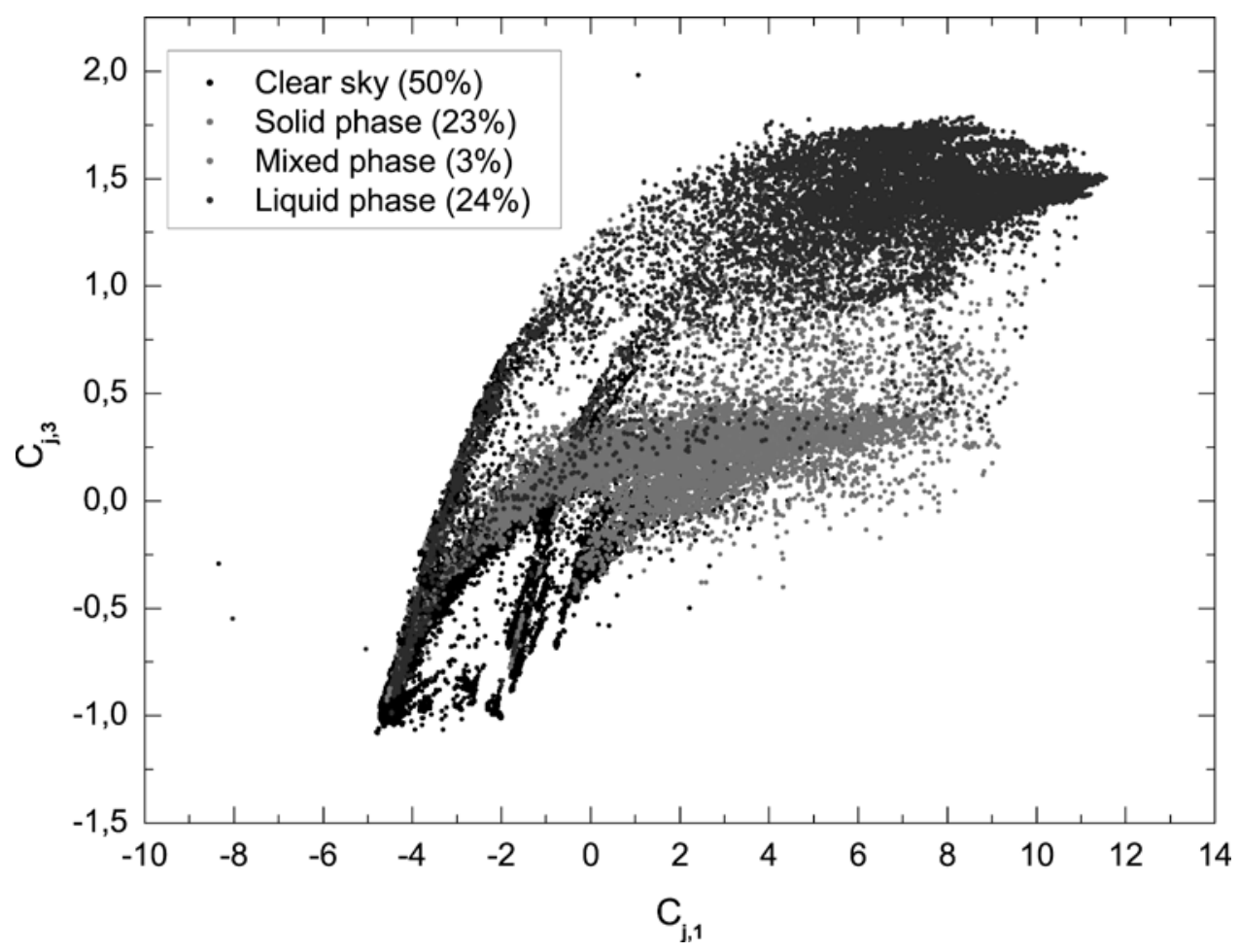

Figure 3. Same as Figure 2. Here, the cloud classification has been obtained from direct PMS probe measurements. The black data points represent clear sky measurements. See color version of this figure at back of this issue.

validation of the classification technique presented in section 2.3 , as well as to assess the information content of the polar nephelometer measurements.

[27] To study this issue, the iterative inversion method developed by Oshchepkov et al. [2000], using physical modeling of the scattered light, is applied to the average scattering phase functions within a given class. We recall here that the method is based on a bi-component (water droplet and ice crystal) representation of cloud composition and uses the non-linear least square fitting of the scattering phase function using smoothness constraints on the desired particle size distributions (PSD). Measurement errors at each angle and PSD's values for each size, in a sense of probability density function, are assumed to be described by the lognormal law, which is the most natural way to take a priori information about the non-negativity of these quantities [Tarantola, 1994]. Note that no analytical expression for the particle size distribution is assumed for the converging solution in this method. The only constraint in this connection is smoothness, needed to avoid an unrealistic jagged structure of the desired size distribution, because the inverse problem is ill posed without constraints.

[28] The inversion method is designed for the retrieval of two volume particle equivalent size distributions simultaneously, one for ice crystals and another for (spherical) water droplets. We need, however, to specify a lookup table containing scattering phase functions of individual ice crystals. In this paper, we have considered only hexagonal ice crystals with different aspect ratios and randomly oriented in 3D space. The scattering phase function of spherical water droplets follows from classic Lorenz-Mie theory and the scattering patterns of hexagonal crystals are computed by an improved geometric-optics model [Yang and Liou, 1996]. In the setting up of the lookup table, we define an equivalent size of ice crystal through the radius of an area-equivalent circle whose area is equal to the ice crystal's cross-section for random orientation.

[29] The inversion results (in terms of particle size distribution) along with direct measurements (from PMS FSSP-100 and 2D-C probes), for the three typical types of clouds (water cloud (a), mixed-phase cloud (b) and ice cloud (c)) are displayed on the lower panels of Figure 4. The retrievals are obtained for ice crystal aspect ratio equal to unity. In the upper panel of this figure, the corresponding initial scattering phase functions are also shown. The mean measured scattering phase functions, defined according to the classification defined on Figure 2, are plotted here along with theoretical ones obtained according to the retrievals for each water and ice component. The vertical error bars on the measured scattering phase functions represent the uncertainties caused by both instrumental errors of measurements and variation of the microphysical properties of the clouds. They are defined through diagonal elements of the corresponding covariance matrix obtained for each selected type of cloud (section 3). The results can be summarized as follows:

1. For water clouds (Figure 4a), the results show very good agreement between the direct measurements and retrieved ones as already discussed by Gayet et al. [2002]. The contribution of ice crystals being very small, the value of the retrieved ice size-particle distribution is negligible in comparison with the water-droplet size distribution. The retrieved scattering phase function is almost equal to the one corresponding to the water contribution.

2. In the mixed phase case (Figure $4 b$ ), the retrieved results of both particle size distributions (water droplets and 
(a) Water
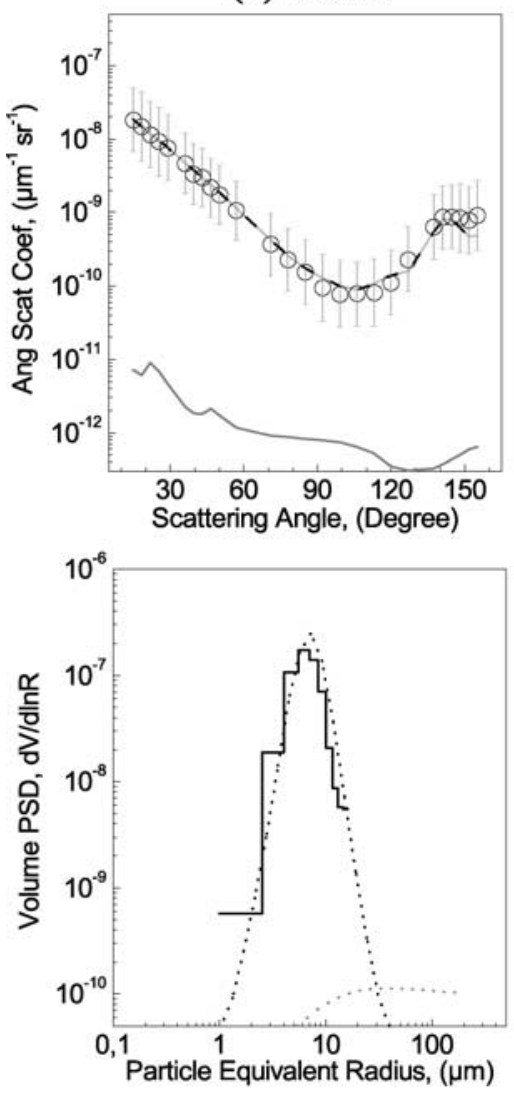

(b) Mixed-Phase
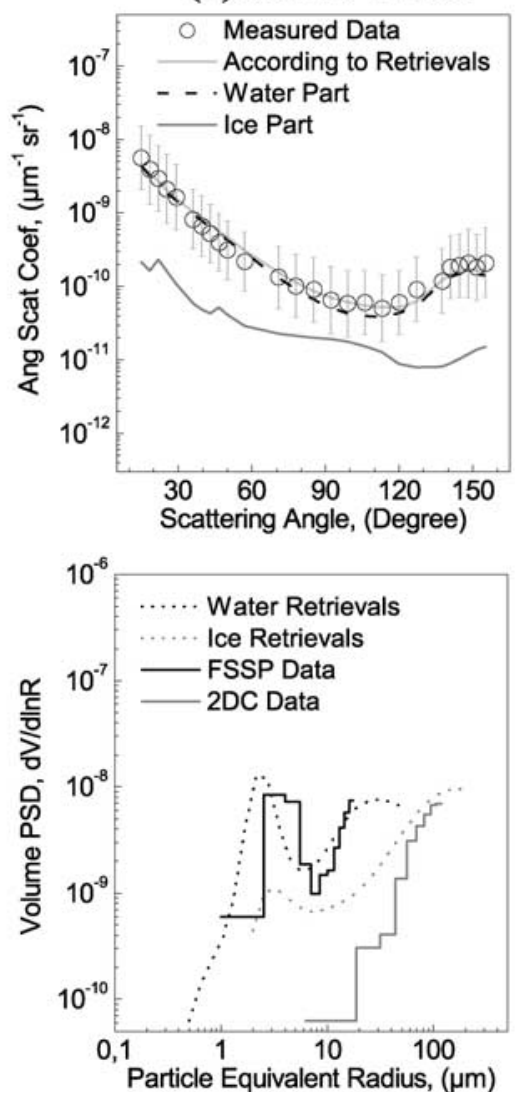

(c) Ice
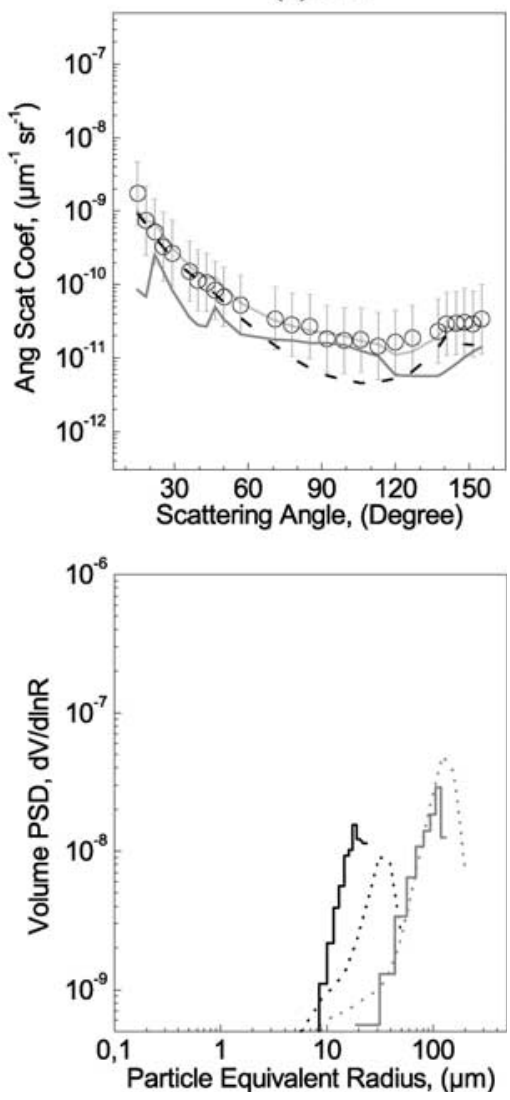

Figure 4. Inversion of the averaged scattering phase functions for three types of cloud: (a) Water droplet cloud, (b) Mixed-phase cloud and (c) Ice crystals clouds. Upper panel: Measured and retrieved scattering phase functions. The contributions on scattering properties are displayed for both particle compositions (water and ice). Bottom panel: Direct (PMS probes) and retrieved particle size distributions.

ice crystals) are also in reasonably good agreement with the direct measurements. Although the water droplet contribution dominates the scattering features of the cloud, the contribution of ice particles is sensitive enough, to be detected with the inversion technique particularly in the side scattering angles [Oshchepkov et al., 2000].

3 . The presence of a non-negligible so-called 'water component' in ice clouds (Figure 4c) represents small supercooled droplets or spherical ice particles, as discussed by Oshchepkov and Isaka [1997] for laboratory measurements. The retrieved ice particle size distribution agrees well with the direct observations.

[30] The above results show that polar nephelometer measurements (between $15^{\circ}$ and $155^{\circ}$ ) contain a considerable amount of information on cloud composition, which can be traced to the systematic differences in scattering patterns for water droplets and ice crystals. The ability of the inversion technique to discriminate the contribution of the components leads to the retrieval of representative particle size distributions.

\section{Conclusions and Outlook}

[31] The main goal of this study is to present typical cloud optical properties in terms of scattering phase functions from $15^{\circ}$ to $155^{\circ}$ along with microphysical parameters (particle size distributions) for three specific types of clouds relative to their phase (liquid-water droplets, mixed-phase and ice-particles). A large set of in situ measurements were analyzed using a principal component analysis. The classification of the revealed patterns leads to draw up three typical averaged scattering phase functions measured by the polar nephelometer along with the corresponding particle size distribution obtained by direct PMS probe measurements. The cloud classification in terms of particle phase composition (water droplets, mixed-phase and ice crystals) is achieved by using a neural network (multilayer perceptron), which has the advantage of involving the equivalent of 28 parameters (a scattering phase function on 28 scattering angles) to characterize the microphysical and optical properties of cloud particles. This classification is validated by the discrimination of the cloud water phase on the basis of the ratio of bulk microphysical parameters derived from direct PMS probe measurements.

[32] The interpretation of the results using an inversion technique show that the information contained in the scattering phase function measurements is sufficient to restore component composition and particle size distribution. This statement is supported by rather a good agreement of the inversion results with the particle size composition obtained by the direct PMS probe measurements for each selected type of cloud.

[33] In the near future, we will report on our implementation of the extrapolation of the scattering phase function 
for small forward and backward angles. This is done by computing the scattering behavior of the retrieved size distribution. Furthermore, this will be extended to nearinfrared wavelengths. These phase functions could eventually be included in radiative transfer analyses to achieve reliable retrievals of the microphysical and optical properties of clouds that matter in climate studies.

[34] Acknowledgments. We are very grateful to J. F. Fournol, C. Duroure, F. Auriol, O. Crépel and L. Cortès for their helpful collaboration in the field experiments. The ARAT'97 experiment was funded under grants from DRET contract 9634104 and from the PATOM Committee (CNRS/INSU). The CIRRUS'98 experiment was supported by the French DSP/STTC (Direction des systèmes de forces et de la Prospective/Service Technique des Technologies Communes) within the ONERA contract 23.140/DA.B1/BC. The JACCS program is supported by the Science and Technology Agency of Japanese Government. The authors thank Anthony Davis for his help in improving the manuscript.

\section{References}

Asano, S., A. Uchiyama, A. Yamazaki, J. F. Gayet, and M. Tanizono, Two case studies of winter continental-type water and mixed-phased stratocumuli over the sea, 2, Absorption of solar radiation, J. Geophys. Res., 107(D21), doi:10.1029/2001JD001108, 2002.

Auriol, F., J. F. Gayet, G. Febvre, O. Jourdan, L. Labonnote, and G. Brogniez, In situ observations of cirrus cloud scattering phase function with $22^{\circ}$ and $46^{\circ}$ halos: Cloud field study on February 1998, J. Atmos. Sci., $58,3376-3390,2001$.

Cober, S. G., G. A. Isaac, A. V. Korolev, and J. W. Strapp, Assessing cloudphase conditions, J. Appl. Meteorol., 40, 1967-1983, 2001

Doutriaux-Boucher, M., J. C. Buriez, G. Brogniez, and L. C. Labonnote, Sensitivity of retrieved POLDER directional cloud optical thickness to various ice particle models, Geophys. Res. Lett., 27, 109-112, 2000.

Durand, G., J. F. Gayet, L. Kaës, and P. Matharan, Airborne infrared and microphysical measurements on cirrus clouds, in Satellite Remote Sensing of Clouds and the Atmosphere III, Proc. SPIE Int. Soc. Opt. Eng., 3495, 72-81, 1998.

Duroure, C., F. Auriol, O. Crepel, J.-F. and Gayet, Microscale inhomogeneities study using high resolution polar nephelometer measurements, paper presented at XXIII General Assembly, Eur. Geophys. Soc., Nice, France, 20-24 April 1998.

Gayet, J. F., F. Auriol, S. L. Oshchepkov, F. Schröder, C. Duroure, G. Febvre, J. F. Fournol, O. Crépel, P. Personne, and D. Daugeron, In situ optical and microphysical measurements with a new airborne "polar nephelometer,", Geophys. Res. Lett., 25, 971-974, 1998.

Gayet, J. F., S. Asano, A. Yamazaki, A. Uchiyama, A. Sinyuk, O. Jourdan, and F. Auriol, Two case studies of continental-type water and maritime mixed-phased stratocumuli over the sea, 1, Microphysical and optical properties, J. Geophys. Res., 107(D21), 1, doi:10.1029/2001JD001106, 2002.

Johnson, R. A., and D. J. Wichern, Applied Multivariate Statistical Analysis, 4th ed., 816 pp., Prentice-Hall, Old Tappan, N. J., 1998.

Kinne, S., T. P. Ackerman, A. J. Heymsfield, F. P. J. Valero, K. Sassen, and J. D. Spinhirne, Cirrus microphysics and radiative transfer: Cloud field study on 28 October 1986, Mon. Weather Rev., 20, 661-684, 1992.

Legendre, P., and L. Legendre, Numerical Ecology, 2nd English ed., 853 pp., Elsevier Sci., New York, 1998.

MacKay, D. J. C., A practical Bayesian framework for back-propagation networks, Neural Comput., 4, 589-603, 1992.

Oshchepkov, S. L., and H. Isaka, Inverse scattering problem for mixed-phase and ice clouds, part 1, Numerical simulation of particle sizing from scattering phase function measurements, Appl. Opt., 36, 8765-8774, 1997.

Oshchepkov, S. L., H. Isaka, J. F. Gayet, A. Sinyuk, F. Auriol, and S. Havemann, Microphysical properties of mixed-phase and ice clouds retrieved from in situ airborne "polar nephelometer" measurements, Geophys. Res. Lett., 27, 209-213, 2000.

Rossow, W. B., and R. A. Schiffer, ISCCP cloud data products, Bull. Am. Meteorol. Soc., 72, 2-20, 1991.

Tarantola, A., Inverse Problem Theory: Model for Data Fitting and Model Parameter Estimation, 1st ed., 601 pp., Elsevier Sci., New York, 1994.

Yang, P., and K. N. Liou, Geometric-optics-integral-equation method for light scattering by nonspherical ice crystals, Appl. Opt., 35, 6568-6584, 1996.

J.-F. Gayet, H. Isaka, O. Jourdan, and S. Oshchepkov, Laboratoire de Météorologie Physique, UMR/CNRS 6016, Université Blaise Pascal, 24, avenue des Landais, 63177 Aubiere Cedex, France. (jourdan@opgc.univbpclermont.fr)

V. Shcherbakov, Stepanov Institute of Physics, Skariny av. 68, Minsk, Belarus 220072. 


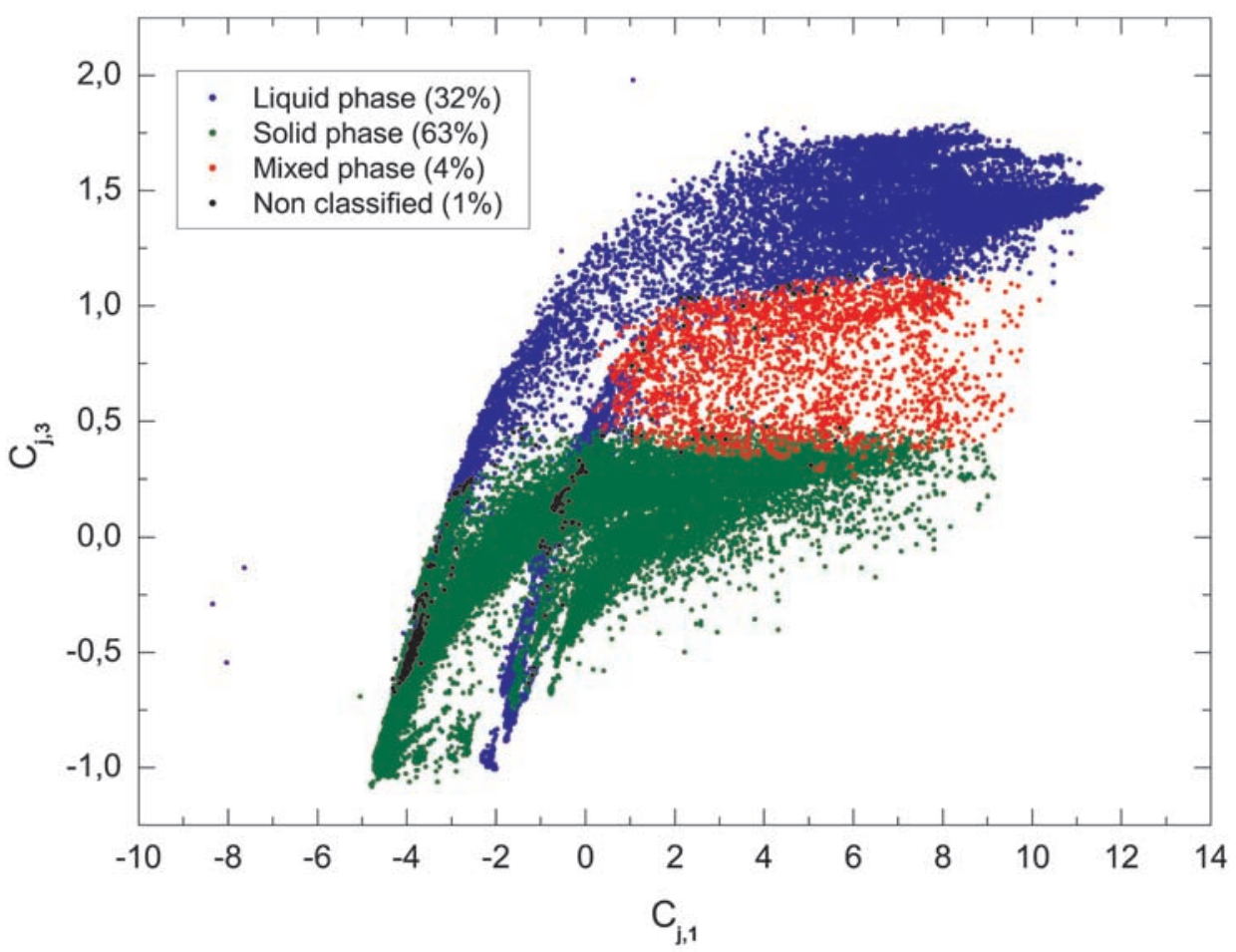

Figure 2. Expansion coefficient diagram: First coefficient $\left(\mathrm{C}_{\mathrm{j}, 1}\right)$ versus the third coefficient $\left(\mathrm{C}_{\mathrm{j}, 3}\right)$. The clusters are colored (blue, red and olive green) according to the cloud composition (water droplet clouds, mixed-phase clouds and ice particles respectively).

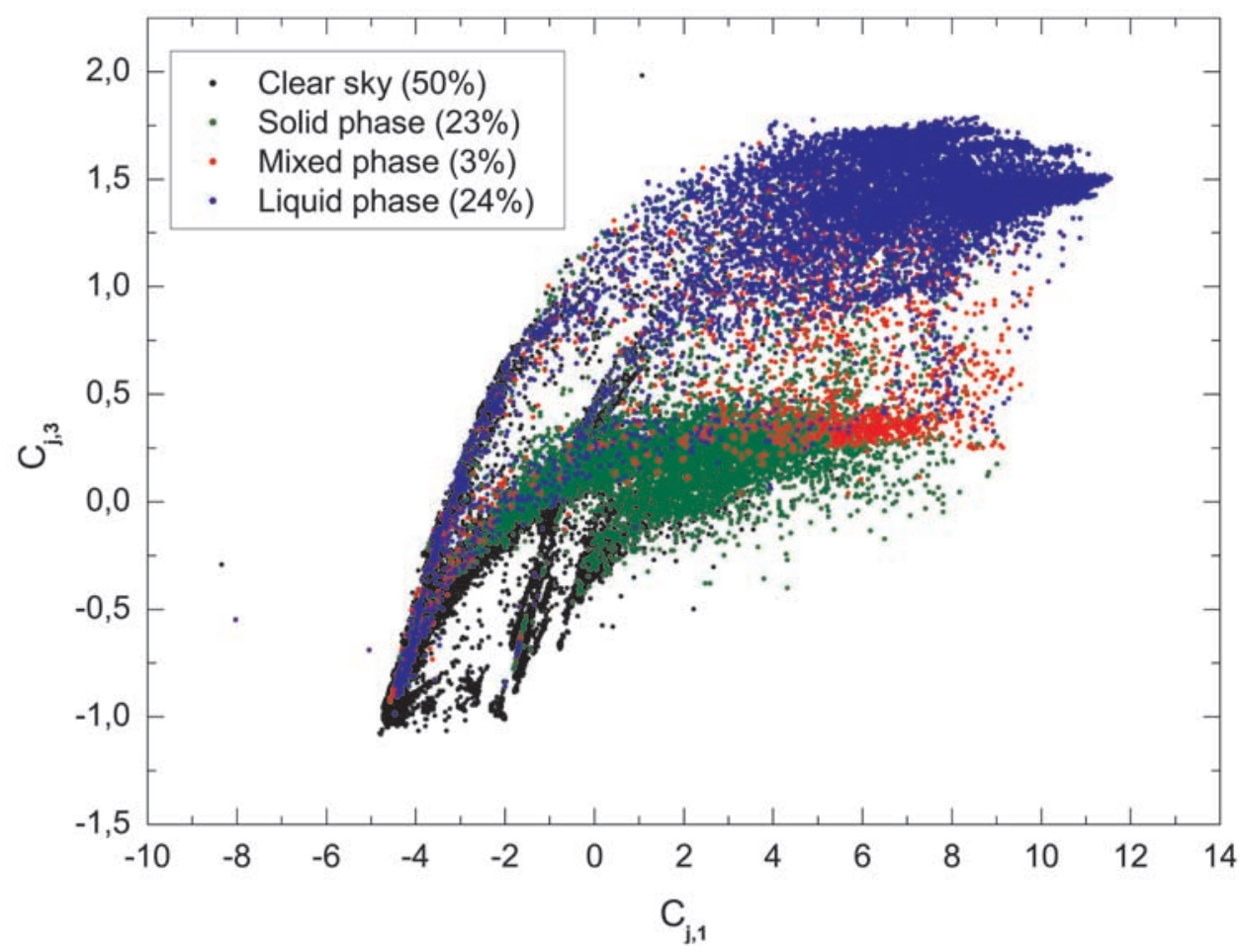

Figure 3. Same as Figure 2. Here, the cloud classification has been obtained from direct PMS probe measurements. The black data points represent clear sky measurements. 